Pensamiento Crítico N. ${ }^{\circ} 15$, pp. 7-22

\title{
La economía china en la coyuntura económica mundial y las implicancias para el Perú
}

Carlos Aquino Rodríguez

\section{RESUMEN}

China ha estado creciendo a altas tasas los últimos años y se está convirtiendo en el motor de la economía mundial. En la crisis económica mundial del 2009 el gobierno chino aplicó políticas para evitar que esa crisis lo afecte. Se espera que frente al panorama incierto de la economía mundial del 2012 China siga sosteniendo a la economía mundial y en esa medida el Perú, que tiene a China ya como su mayor socio comercial, no se vea afectado tanto por esta coyuntura.

Palabras clave: China, economía mundial, exportaciones, Perú.

\begin{abstract}
China has been growing at high rates in the last years and it is becoming the engine of the world economy. In the 2009 world economic crisis the Chinese government took several economic policies that allowed it to avoid been affected. Given the uncertainties surrounding the world economy in the year 2012 it is expected that China will also grow fast and sustain the world economy. By doing this it will help the Peruvian economy given the fact that China has become already our top trading partner.
\end{abstract}

Keywords: China, World economy, Perú exports 


\section{Pensamiento Crítico N. 15}

\section{Introducción}

Con la economía de los países avanzados como Europa y EE.UU. en incertidumbre respecto a su crecimiento, especialmente en Europa, donde es probable no haya crecimiento el 2012, y con la economía japonesa en problemas también, el mundo mira a China para evitar caer en un estancamiento o en una recesión nuevamente, como sucedió en el 2009. Ese año la economía mundial decreció un $-0.7 \%$ y no más a pesar de que las economías avanzadas decrecieron un -3.7\%. Lo que pasó fue que las economías emergentes y en desarrollo crecieron ese año $2.8 \%$, y China en particular creció 9.2\% (Ver Cuadro 1).

China es la segunda economía más grande del mundo, el mayor exportador, y el que tiene las mayores reservas internacionales, que actualmente están en 3.2 billones de dólares. Además en los primeros siete meses del año 2011 China se convirtió en el mayor mercado para las exportaciones del Perú.

En este artículo se analizará cual es la situación actual de la economía china, cómo pudo capear la crisis del 2009, cómo podría afrontar otra crisis en el 2012 y como conclusiones, cuales son las implicancias para el mundo, especificamente para el perú.

\section{Situación actual de la economía china}

Como se indicó en el Cuadro 1 (y en el Cuadro 2 más actualizado), el excepcional crecimiento de China y los países emergentes, como la India en el 2009, evitaron que el mundo caiga en una recesión mayor ese año pese a la caída abultada de las economías avanzadas. China creció un $9.2 \%$ ese año, pero esto es una tendencia que el país observa desde hace más de treinta años.

Desde que China empezó sus reformas económicas en 1979, el país ha crecido por 32 años consecutivos a una tasa media anual de $10 \%$, algo sin paralelo en la historia mundial. Ahora, esto no debería sorprender mucho si se conoce algo de la historia de China y de su proceso de desarrollo económico. 


\section{Carlos Aquino Rodríguez}

Cuadro 1. Crecimiento de la economía mundial y de los principales países Table 1.1. Overview of the World Economic Outlook Projections (Percent change unless noted otherwise)

Year over Year

\begin{tabular}{|l|c|c|c|c|}
\hline & 2009 & 2010 & 2011 & 2012 \\
\hline World Output1 & $\mathbf{- 0 . 7}$ & $\mathbf{5 . 1}$ & $\mathbf{4 . 0}$ & $\mathbf{4 . 0}$ \\
\hline Advanced Economies & $\mathbf{- 3 . 7}$ & $\mathbf{3 . 1}$ & $\mathbf{1 . 6}$ & $\mathbf{1 . 9}$ \\
\hline United States & -3.5 & 3.0 & 1.5 & 1.8 \\
\hline Euro Area & -4.3 & 1.8 & 1.6 & 1.1 \\
\hline Germany & -5.1 & 3.6 & 2.7 & 1.3 \\
\hline France & -2.6 & 1.4 & 1.7 & 1.4 \\
\hline Italy & -5.2 & 1.3 & 0.6 & 0.3 \\
\hline Spain & -3.7 & -0.1 & 0.8 & 1.1 \\
\hline Japan & -6.3 & 4.0 & -0.5 & 2.3 \\
\hline United Kingdom & -4.9 & 1.4 & 1.1 & 1.6 \\
\hline Canada & -2.8 & 3.2 & 2.1 & 1.9 \\
\hline Other Advanced Economies & -1.1 & 5.8 & 3.6 & 3.7 \\
\hline Newly Industrialized Asian Economies & -0.7 & 8.4 & 4.7 & 4.5 \\
\hline Emerging and Developing Economies & $\mathbf{2 . 8}$ & $\mathbf{7 . 3}$ & $\mathbf{6 . 4}$ & $\mathbf{6 . 1}$ \\
\hline Central and Eastern Europe & -3.6 & 4.5 & 4.3 & 2.7 \\
\hline Commonwealth of Independent States & -6.4 & 4.6 & 4.6 & 4.4 \\
\hline Russia & -7.8 & 4.0 & 4.3 & 4.1 \\
\hline Excluding Russia & -3.0 & 6.0 & 5.3 & 5.1 \\
\hline Developing Asia & 7.2 & 9.5 & 8.2 & 8.0 \\
\hline China & 9.2 & 10.3 & 9.5 & 9.0 \\
\hline India & 6.8 & 10.1 & 7.8 & 7.5 \\
\hline ASEAN-5* & 1.7 & 6.9 & 5.3 & 5.6 \\
\hline Latin America and the Caribbean & -1.7 & 6.1 & 4.5 & 4.0 \\
\hline Brazil & -0.6 & 7.5 & 3.8 & 3.6 \\
\hline Mexico & -6.2 & 5.4 & 3.8 & 3.6 \\
\hline Middle East and North Africa & 2.6 & 4.4 & 4.0 & 3.6 \\
\hline Sub-Saharan Africa & 2.8 & 54 & 5.2 & 5.8 \\
\hline Memorandum & & & & \\
\hline European Union & -4.2 & 1.8 & 1.7 & 1.4 \\
\hline
\end{tabular}

* Indonesia, Malaysia, Philippines, Thailand, and Vietnam.

Fuente: World Economic Outlook, setiembre 2011, FMI, cuadro 1 http://www.imf.org/external/pubs/ft/ weo/2011/02/index.htm 


\section{Pensamiento Crítico N. I5}

Cuadro 2. Economía mundial: Resumen de las proyecciones

\begin{tabular}{|c|c|c|c|c|c|c|c|c|c|c|}
\hline & 2007 & 2008 & 2009 & 2010 & 2011 & 2012 & 2013 & 2014 & 2015 & 2016 \\
\hline \multicolumn{11}{|l|}{ Real GDP growth (\%) } \\
\hline World (PPP exchange rates) a & 5.2 & 2.6 & -0.9 & 4.9 & 3.7 & 3.3 & 4.0 & 4.1 & 4.3 & 4.3 \\
\hline $\begin{array}{l}\text { World (market exchange } \\
\text { rates) }\end{array}$ & 3.9 & 1.4 & -2.5 & 3.9 & 2.5 & 2.1 & 2.8 & 2.9 & 3.1 & 3.1 \\
\hline US & 1.9 & -0.3 & -3.5 & 3.0 & 1.6 & 1.3 & 1.9 & 2.2 & 2.4 & 2.3 \\
\hline Japan & 2.3 & -1.2 & -6.3 & 4.0 & -0.3 & 2.3 & 1.3 & 1.3 & 1.3 & 1.4 \\
\hline Euro area & 2.9 & 0.3 & -4.2 & 1.7 & 1.6 & -0.3 & 1.1 & 1.5 & 1.6 & 1.7 \\
\hline China & 14.2 & 9.6 & 9.2 & 10.4 & 9.0 & 8.2 & 8.3 & 8.1 & 8.1 & 8.0 \\
\hline Eastern Europe & 7.5 & 4.5 & -5.6 & 3.3 & 3.6 & 3.3 & 3.9 & 4.0 & 4.0 & 4.2 \\
\hline $\begin{array}{l}\text { Asia \& Australasia (excl } \\
\text { Japan) }\end{array}$ & 9.3 & 5.7 & 5.1 & 8.3 & 6.6 & 6.5 & 6.7 & 6.6 & 6.7 & 6.5 \\
\hline Latin America & 5.6 & 4.0 & -2.1 & 5.9 & 3.7 & 3.5 & 4.2 & 4.3 & 4.1 & 4.2 \\
\hline Middle East \& North Africa & 5.0 & 5.3 & 1.5 & 4.2 & 3.1 & 4.0 & 4.5 & 4.9 & 4.7 & 4.9 \\
\hline Sub-Saharan Africa & 7.0 & 4.9 & 1.2 & 4.4 & 4.4 & 5.0 & 4.8 & 4.6 & 4.9 & 5.0 \\
\hline World inflation (\%; av) & 3.4 & 4.9 & 1.6 & 3.0 & 3.8 & 3.1 & 3.2 & 3.2 & 3.1 & 3.2 \\
\hline World trade growth (\%) & 7.1 & 2.8 & -12.0 & 14.1 & 6.8 & 5.2 & 6.1 & 6.4 & 6.6 & 6.5 \\
\hline \multicolumn{11}{|l|}{ Commodity prices } \\
\hline Oil (US\$/barrel; Brent) & 72.71 & 97.66 & 61.86 & 79.63 & 110.00 & 90.00 & 95.00 & 100.00 & 104.00 & 110.00 \\
\hline $\begin{array}{l}\text { Industrial raw materials } \\
\text { (US\$; \% change) }\end{array}$ & 11.3 & -5.3 & -25.6 & 45.4 & 23.7 & -10.1 & 1.3 & -4.0 & 0.6 & 3.0 \\
\hline $\begin{array}{l}\text { Food, feedstuffs \& beverages } \\
\text { (US\$; \% change) }\end{array}$ & 30.9 & 28.1 & -20.3 & 10.7 & 31.4 & -11.5 & -8.1 & 1.1 & 3.1 & 2.4 \\
\hline \multicolumn{11}{|l|}{$\begin{array}{l}\text { Exchange rates (annual } \\
\text { av) }\end{array}$} \\
\hline$¥:$ US\$ & 118 & 103 & 94 & 88 & 80 & 77 & 80 & 82 & 84 & 82 \\
\hline US\$:€ & 1.37 & 1.47 & 1.39 & 1.33 & 1.39 & 1.33 & 1.28 & 1.23 & 1.28 & 1.27 \\
\hline
\end{tabular}

Fuente: "World economy: EIU forecast - Downgrading the euro zone and the US", 20 de octubre del 2011, http://viewswire.eiu.com/index.asp?layout=VWArticleVW3\&article_id =348524419\&region id $=1510000351 \&$ refm $=$ vwReg\&page title $=$ Latest + regional + analysis\&fs $=$ true $\&$ mkt tok $=3 \mathrm{RkMMJWWfF} \overline{9}$ wsRons6nJZKXonjHpfsX57\%2BUtWKC̄g38431UFwdcjKPmjr1YEBTcN0dvycMRAVFZ15nQIRD7I\%3D\&rf=0 


\section{Carlos Aquino Rodríguez}

China en estos momentos está recuperando el sitial que tenía en la economía mundial, que perdió en los últimos dos siglos. Se calcula que hacia el año 1820 la economía de China representaba un tercio del PBI mundial (Ver Gráfico 1). Esto se redujo a casi $4 \%$ en 1950 , su punto más bajo.

China atravesó un periodo de declinación económica cuando la Revolución Industrial empieza en Occidente a fines del siglo XVIII y los beneficios de esta hace que los países europeos primero, y EE.UU. después, empiecen a crecer. China perdió estos beneficios pues se había aislado del mundo desde comienzos del siglo XVII. Esto se agudizó cuando fue abierto a la fuerza a mitad del siglo XIX, y sumado a las guerras, revoluciones que sufrió, culmina en 1949 con la revolución comunista de ese año. Pero desde 1979 el país empieza a crecer y recuperar su sitial en el mundo.

Otra razón para que no sorprenda el crecimiento chino es que el crecimiento económico de este país no es único, es un modelo que ya se siguió en el Asia Oriental, con Japón, Corea, Taiwán y otros, que durante más de 20 o 30 años también crecieron a altas tasas. Ese modelo incluyó una intervención del gobierno, protección de su industria nacional y orientación al exterior.

Gráfico 1. Participación de China y algunos países en la economía mundial

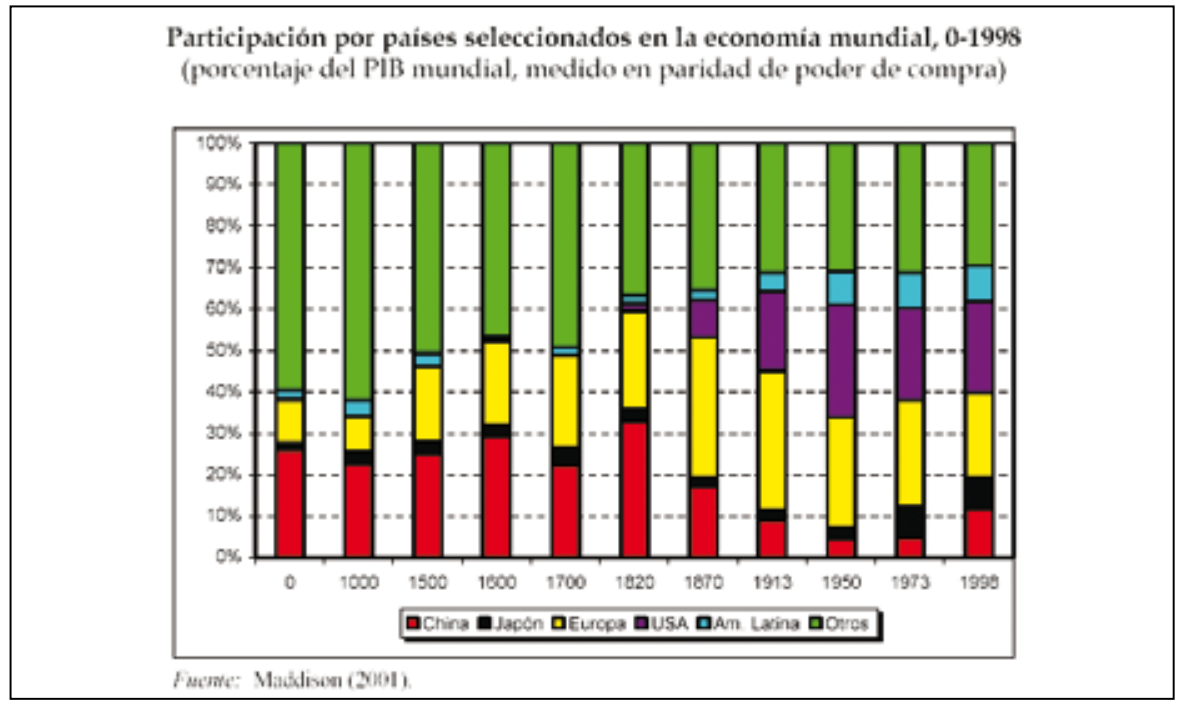

Fuente: "El surgimiento de China: Una visión desde América Central", Manuel R. Agosin, y otros http://www.iadb.org/regions/re2/surgimiento\%20china\%2004-10-29.pdf 


\section{Pensamiento Crítico N. ${ }^{\circ}$}

Gráfico 2. Las tres mayores potencias económicas: 1870, 1973, 2010, 2030

Top three countries by economic dominance

$\%$ share* of global economic power
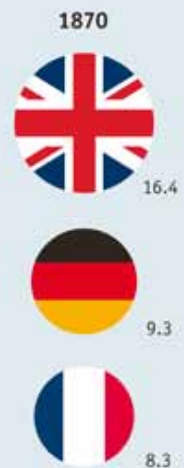

Source: Arvind Subramanian
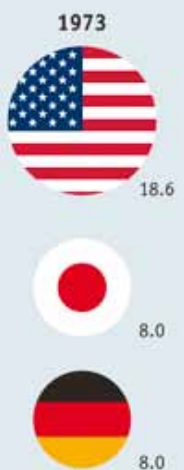

8.0

Fuente:http://www.economist.com/blogs/dailychart/2011/09/global-economic-dominance

Por último, China tiene casi un quinto de la población mundial, la mayor en el mundo, así que el tamaño de su mercado, de su población, le posibilita crecer más tiempo que los demás países asiáticos y llegar a ser la mayor economía en el mundo.

Todo esto permitió que China se haya convertido actualmente en la segunda mayor economía del mundo, con un Producto Bruto Interno (PBI) de casi 5.9 billones de dólares en el 2010, un aumento de casi 5 veces respecto del 2000, como se ve en Cuadro 2, y que su PBI per cápita haya pasado de 949 dólares a 4392 dólares en ese mismo lapso (un aumento de 4,6 veces).

A la velocidad que crece China, y según lo pronostican muchas instituciones, en el lapso de una década podrá superar a EE.UU. convirtiéndose en la mayor economía mundial $^{1}$ (Ver Gráfico 2).

El crecimiento económico chino ha sido impulsado por las exportaciones, que alcanzaron un nivel de $35 \%$ de su economía en el 2008, para caer a un $26,7 \%$ de su PBI en el 2009 por la crisis económica mundial de ese año (exportaciones de bienes y servicios, Cuadro 2).

1 "Becoming number one: China's economy would overtake America's within a decade"”, The Economist, setiembre 24, 2011, http://www.economist.com/node/21528987 
Carlos Aquino Rodríguez

\begin{tabular}{|c|c|c|c|c|c|c|c|c|c|c|}
\hline 옹 & ִֻ & $\begin{array}{l}0 \\
\infty \\
\infty \\
\infty \\
\infty\end{array}$ & ڤั. & 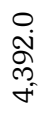 & 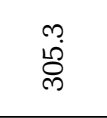 & $\begin{array}{l}\infty \\
\omega \\
\omega\end{array}$ & 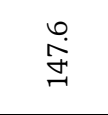 & 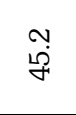 & $\begin{array}{l}\hat{n} \\
\tilde{\sigma} \\
\hat{N}\end{array}$ & 임 \\
\hline 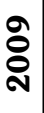 & ஸे & $\begin{array}{l}\text { Na } \\
\text { ğ }\end{array}$ & $\stackrel{\sim}{\sim}$ & $\begin{array}{r}\stackrel{N}{+} \\
\stackrel{\infty}{N} \\
\dot{n}\end{array}$ & ت. & ڤึ & 㟔 & $\stackrel{5}{\sim}$ & 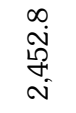 & $\begin{array}{l}0 \\
\dot{1} \\
\dot{10}\end{array}$ \\
\hline $\begin{array}{l}\infty \\
\stackrel{8}{8} \\
\text { N }\end{array}$ & $\begin{array}{l}\ddot{a} \\
\text {. }\end{array}$ & $\begin{array}{l}\infty \\
\stackrel{\infty}{N} \\
\stackrel{\sim}{i} \\
+\end{array}$ & $\begin{array}{l}\text { ㅇ. } \\
\text { ले }\end{array}$ & $\begin{array}{l}0 \\
\dot{m} \\
\overrightarrow{+} \\
\dot{m}\end{array}$ & $\begin{array}{l}\stackrel{\overbrace{}}{\mathrm{H}} \\
\stackrel{\gamma}{F}\end{array}$ & חִ & $\begin{array}{l}\infty \\
\stackrel{\sim}{\sim} \\
\stackrel{\sim}{\sim}\end{array}$ & $\stackrel{\circ}{\dot{Z}}$ & 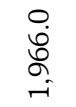 & นْ \\
\hline 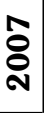 & $\begin{array}{l}\underset{+}{+} \\
\text {. }\end{array}$ & $\begin{array}{l}0 \\
\dot{+} \\
\text { \& } \\
\text { m }\end{array}$ & $\begin{array}{l}\stackrel{+}{\infty} \\
\infty \\
\infty\end{array}$ & $\begin{array}{l}\text { m } \\
\text { in } \\
\text { i }\end{array}$ & 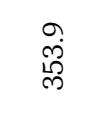 & $\stackrel{\sim}{\sim}$ & $\underset{\stackrel{\infty}{\sim}}{\stackrel{\infty}{\sim}}$ & 각 & 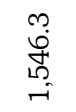 & $\begin{array}{l}+ \\
\text { N่ }\end{array}$ \\
\hline 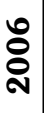 & $\stackrel{\widetilde{\sim}}{\stackrel{\sim}{\sim}}$ & 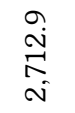 & ڤ̆ं & $\begin{array}{l}\text { ma } \\
\text { gु } \\
\text { ò } \\
\text { ì }\end{array}$ & సิ่ & $\overrightarrow{6}$ & $\begin{array}{l}\stackrel{\sim}{\infty} \\
\stackrel{\oplus}{\sim}\end{array}$ & $\stackrel{\circ}{\stackrel{\leftrightarrow}{ }}$ & $\begin{array}{l}\text { ․ } \\
\infty \\
0 \\
0\end{array}$ & $\begin{array}{l}\text { กొ } \\
\text { N่ }\end{array}$ \\
\hline $\begin{array}{l}10 \\
\text { ○̊ } \\
\text { లి }\end{array}$ & $\stackrel{n}{=}$ & 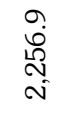 & $\stackrel{\vec{m}}{\text { m }}$ & 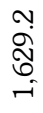 & $\begin{array}{l}\circ \\
\stackrel{\text { mे }}{-}\end{array}$ & $\underset{\omega}{0}$ & ڤึ & $\stackrel{\breve{y}}{\stackrel{H}{*}}$ & $\underset{\infty}{\stackrel{+}{\mid}}$ & $\stackrel{\sim}{g}$ \\
\hline 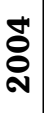 & ㄹ. & 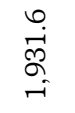 & $\begin{array}{l}\text { ○े } \\
\text { ले }\end{array}$ & 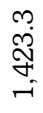 & $\begin{array}{l}0 \\
\infty \\
0\end{array}$ & $\mathscr{L}^{0}$ & 定 & $\begin{array}{l}\mathscr{P} \\
\ddot{q}\end{array}$ & $\begin{array}{l}\text { స్ } \\
\text { }\end{array}$ & $\begin{array}{l}\infty \\
\dot{\varphi} \\
\dot{\gamma}\end{array}$ \\
\hline $\begin{array}{l}\text { ? } \\
\text { ஜ } \\
\text { N }\end{array}$ & $\begin{array}{l}\infty \\
\infty \\
\infty\end{array}$ & $\begin{array}{l}\text { a } \\
\text { ஓं } \\
\text { bु }\end{array}$ & $\begin{array}{l}\text { ஸे } \\
\text { న }\end{array}$ & 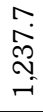 & $\begin{array}{l}\infty \\
\dot{q}\end{array}$ & חִ & 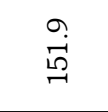 & $\underset{\sim}{\mathscr{Z}}$ & $\begin{array}{l}\overrightarrow{6} \\
\stackrel{7}{7}\end{array}$ & $\stackrel{\circ}{\dot{H}}$ \\
\hline $\begin{array}{l}\text { N } \\
\text { రి } \\
\text { స }\end{array}$ & ä & 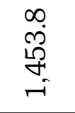 & 岕 & 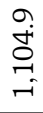 & $\begin{array}{l}\text { में } \\
\text { ले }\end{array}$ & חִ & 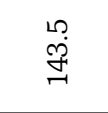 & $\underset{m}{\stackrel{a}{m}}$ & ๙ิ & $\begin{array}{l}\text { m } \\
\text { ó }\end{array}$ \\
\hline ర్రి & $\underset{\infty}{\infty}$ & $\begin{array}{l}\infty \\
\stackrel{\sim}{ \pm} \\
\text { } \\
-i\end{array}$ & $\begin{array}{l}\bullet \\
\stackrel{N}{N}\end{array}$ & $\begin{array}{l}\text { N. } \\
\infty \\
8 \\
8 \\
\end{array}$ & 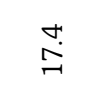 & बे & 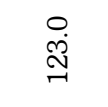 & $\begin{array}{l}\text { nె } \\
\text { లో }\end{array}$ & $\begin{array}{l}\stackrel{0}{0} \\
\stackrel{\text { N }}{\text { N }}\end{array}$ & 官 \\
\hline $\begin{array}{l}\text { § } \\
\text { ○ิ }\end{array}$ & $\stackrel{\leftrightarrow}{\infty}$ & $\begin{array}{l}\stackrel{+}{0} \\
\stackrel{0}{\circ} \\
\stackrel{-}{-}\end{array}$ & $\begin{array}{l}\text { ஸे } \\
\text { Nं }\end{array}$ & ণু & 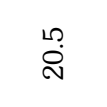 & a) & ને & में & 곡 & $\begin{array}{l}\infty \\
\dot{\rho} \\
\text { ల. }\end{array}$ \\
\hline & 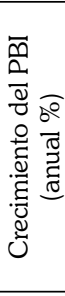 & 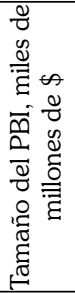 & 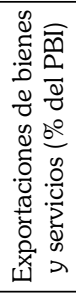 & 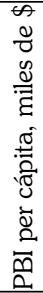 & 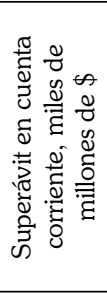 & 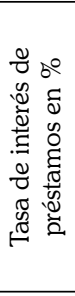 & 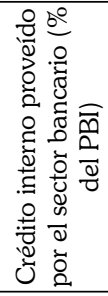 & 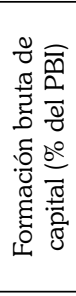 & 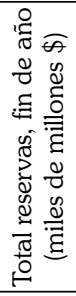 & 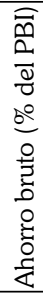 \\
\hline
\end{tabular}




\section{Pensamiento Crítico N. ${ }^{\circ}$}

El otro motor del crecimiento chino es la inversión. China invierte casi el doble que otros países, llegando a un nivel de casi la mitad de su PBI (Ver la formación bruta del capital en el Cuadro 3). Estos dos motores, las exportaciones y la inversión, han crecido en desmedro del otro, el consumo. Como se ve en el Gráfico 2, el consumo de las familias ha decaído persistentemente en los últimos años en China, especialmente desde que el país se abre al mundo en 1979 e implanta un modelo de desarrollo de crecer exportando. En ese periodo, el consumo de las familias ha caído de un nivel de casi $50 \%$ a uno actual de $35 \%$.

Como se ve, en China el consumo es poco, y el ahorro es alto. Según el Cuadro 3 , el ahorro bruto, como porcentaje del PBI, subió de un nivel de $36.8 \%$ en el 2000 a $53.6 \%$ en el 2009 , todo un récord mundial.

Gráfico 3. Consumo de las familias como porcentaje del PBI

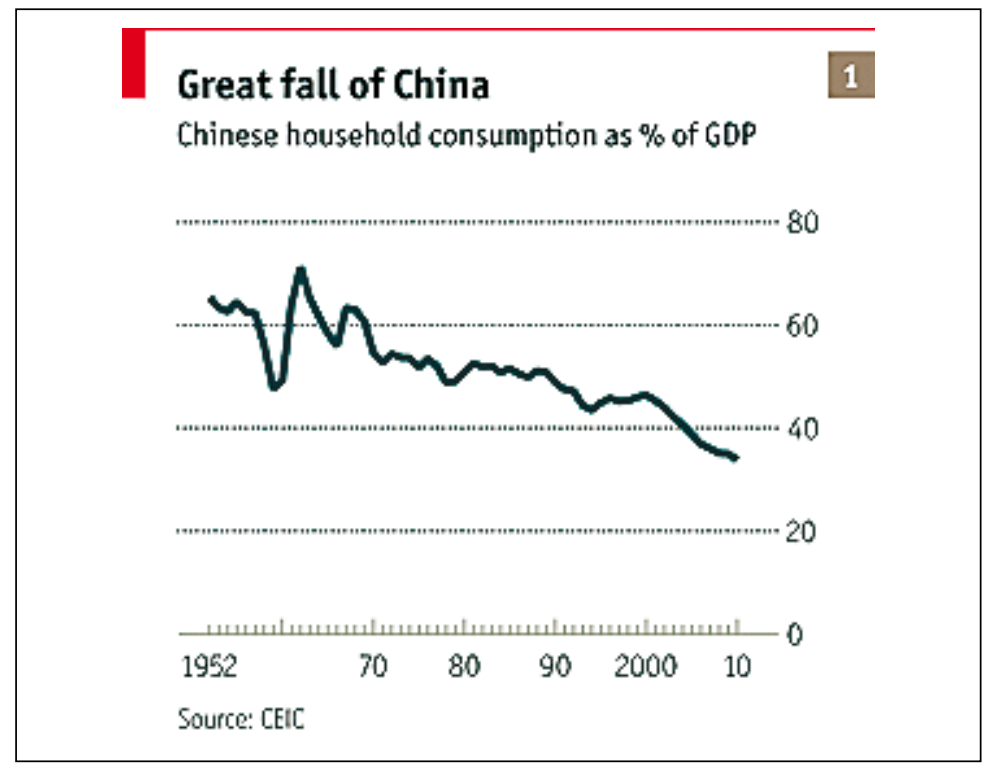

Fuente: http://www.economist.com/node/21528985 


\section{Carlos Aquino Rodríguez}

\section{La economía china y la crisis del 2009}

¿Como China pudo crecer el 2.009 en una alta tasa mientras el mundo caía en recesión?

Como todos los países en el mundo, China el 2009 ante una caída de la economía mundial que vio sus exportaciones caer $16.2 \%$ ese año respecto al anterior (ya se empieza a desacelerar en el 2008 cuando crece solo $17.6 \%$, después de crecer en promedio $25 \%$ por muchos años ${ }^{2}$, apeló a su demanda interna, a través de una rebaja en su tasa de interés para incentivar el consumo y la inversión, y al gasto público.

Como se ve en el Gráfico 4, el gobierno chino redujo la tasa de interés desde el tercer trimestre del 2008 desde un nivel de $7.5 \%$ y lo bajó a enero del 2009 a $5.3 \%$ y lo mantuvo así todo el 2009 y gran parte del 2010, hasta octubre de ese año.

Asimismo, aumentó la oferta monetaria que creció el 2009 un $27.7 \%$ cuando había crecido en los últimos a una tasa promedio de $17 \%$ anual $^{3}$.

Gráfico 4. Tasa de interés, fijado por el Banco Popular de China, en porcentaje (enero 2002 - octubre 2011)

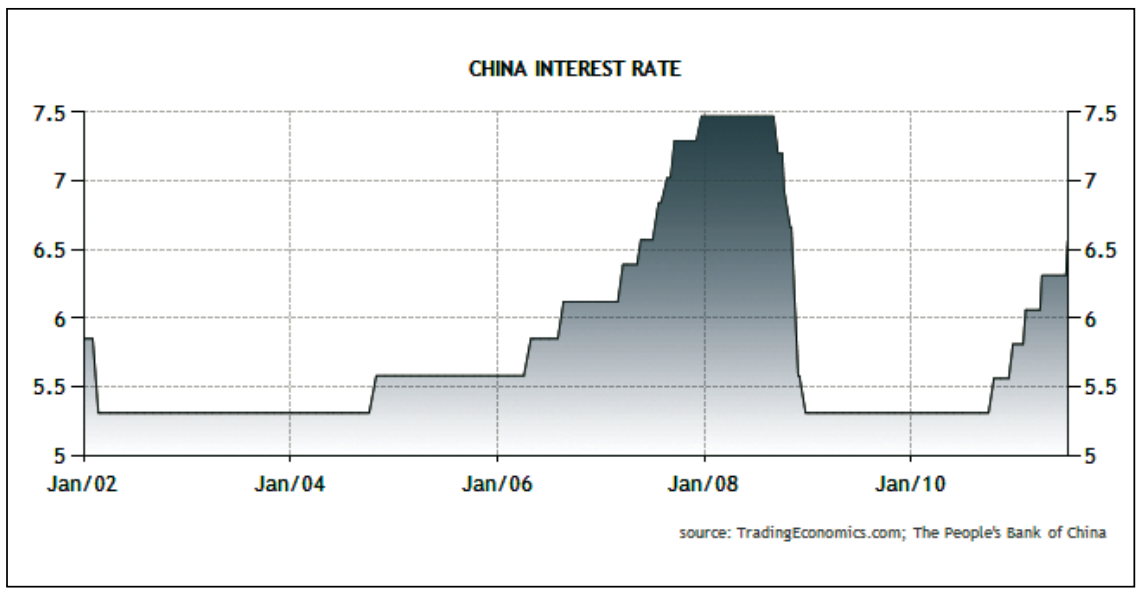

Fuente: http://www.tradingeconomics.com/china/interest-rate

2 Ver Cuadro A10 Apéndice Estadístico: Asian Development Outlook 2011: South-South Economic Links, $\mathrm{http}$ //beta.adb.org/publications/asian-development-outlook-2011-south-south-economic-links

3 Ver Cuadro A9 Apéndice Estadístico. Asian Development Outlook 2011: South-South Economic Links, http://beta.adb.org/publications/asian-development-outlook-2011-south-south-economic-links 


\section{Pensamiento Crítico N. I5}

Así, por ejemplo, la baja en la tasa de interés, y la reducción en el impuesto que se paga por la compra de un auto, aumentó las ventas de estos e hizo que China el 2009 se convierta en el mayor mercado de automóviles en el mundo, al venderse 13.644 millones de autos, un $46.2 \%$ más que en el 2008 . El gobierno chino redujo a la mitad la tasa de impuesto a la venta de autos de $10 \%$ a $5 \%$ y además ofreció 732 millones de dólares en efectivo para que la gente remplace sus autos viejos por nuevos ${ }^{4}$. Esa cifra de autos vendidos el 2009 fue de 1.3 veces mayor del tamaño del mercado de EE.UU.

A fines del año 2008 el gobierno chino dio asimismo un paquete de estímulo fiscal de 4 billones de yuanes (unos 580 mil millones de dólares en ese entones). Este gasto público en China es bastante efectivo pues el gobierno puede gastar rápidamente este dinero en obras pues al tener aún una economía de comando en muchos sectores, cuando el gobierno decide hacer algo, esto se ejecuta rápidamente pues controla todos los poderes del Estado y así la oposición a los proyectos se minimiza.

Así, por ejemplo, los gastos del gobierno central crecieron hasta llegar a representar un $23 \%$ del PBI en el 2009 y en el 2010, después de estar en alrededor del 19\% del $\mathrm{PBI}$ en los tres años anteriores ${ }^{5}$. El gobierno central, que tenía un pequeño superávit el 2007, tuvo un déficit de $-0.4 \%$ del PBI el 2008, de $-2.9 \%$ el 2009, y de $-2.1 \%$ el $2010^{6}$. La inversión bruta de capital aumenta de 41.7\% del PBI en el 2007 a $44 \%$ el 2008, a $47.7 \%$ el 2009 y a $45.2 \%$ del PBI el 2010 (Ver Cuadro 3).

Como se ve en el Cuadro 4 en verdad el motor del crecimiento económico chino, especialmente el año 2009 fue la inversión. Ese año la contribución del Consumo Privado al crecimiento del PBI aumentó, pero no por mucho, mientras que el de la Inversión lo hizo a casi el doble respecto al año anterior.

4 China Ends U.S.'s Reign as Largest Auto Market (Update2) http://www.bloomberg.com/apps/ news?pid = newsarchive \&sid $=\mathrm{aE} \cdot \mathrm{x} \mathrm{r}$ 19NZE

5 Ver Cuadro A21 Apéndice Estadístico: Asian Development Outlook 2011: South-South Economic Links, $\mathrm{http} / / /$ beta.adb.org/publications/asian-development-outlook-2011-south-south-economic-links

6 Ver Cuadro A23 Apéndice Estadístico: Asian Development Outlook 2011: South-South Economic Links, http://beta.adb.org/publications/asian-development-outlook-2011-south-south-economic-links

http://www.bbc.co.uk/news/business-14836386 


\section{Carlos Aquino Rodríguez}

Cuadro 4. Contribución al crecimiento (demanda) (en pocentaje)

\begin{tabular}{|c|c|c|c|c|c|}
\hline Año & Consumo Privado & Consumo de gobierno & Inversión & Exportaciones Netas & PBI \\
\hline 2006 & 3.0 & 1.1 & 5.4 & 3.3 & 12.7 \\
\hline 2007 & 3.8 & 1.6 & 5.6 & 3.3 & 14.2 \\
\hline 2008 & 2.7 & 1.4 & 4.7 & 0.8 & 9.6 \\
\hline 2009 & 2.9 & 1.7 & 8.9 & -4.3 & 9.2 \\
\hline 2010 & 2.8 & 1.1 & 5.6 & 0.8 & 10.3 \\
\hline
\end{tabular}

Fuente: "Asia Development Outlook 2011: South-South Economic Links", April 2011 http://www.adb.org/ documents/books/ado/2011/ado2011-prc.pdf

\section{3. ¿Cómo China podría afrontar otra crisis internacional?}

China actualmente es el segundo mercado más grande del mundo, medido por el tamaño de sus importaciones totales, solo después de EE.UU. El año 2010 EE.UU. importó por 1.968 billones de dólares y China por 1.395 billones (Ver Cuadro 5). Como se ve el mercado chino es solo el $70 \%$ del de EE.UU., aunque crece a un ritmo bastante rápido.

Cuadro 5. Principales exportadores e importadores de mercancías, 2010 (Miles de millones de dólares)

\begin{tabular}{|c|c|c|c|}
\hline Ranking de Países & Monto Exportaciones & Ranking de Países & Monto Importaciones \\
\hline China & 1,578 & EE.UU. & 1,968 \\
\hline EE.UU. & 1,278 & China & 1,395 \\
\hline Alemania & 1,269 & Alemania & 1,067 \\
\hline Japón & 770 & Japón & 693 \\
\hline Holanda & 572 & Francia & 606 \\
\hline Francia & 521 & Inglaterra & 558 \\
\hline Corea & 466 & Holanda & 517 \\
\hline Italia & 448 & Italia & 484 \\
\hline Bélgica & 411 & Hong Kong & 442 \\
\hline Inglaterra & 405 & Corea & 425 \\
\hline
\end{tabular}

Fuente: Apéndice, Cuadro 3, http://www.wto.org/english/res_e/booksp_e/anrep_e/world_trade_report11_e. pdf 


\section{Pensamiento Crítico N. ${ }^{\circ}$ I5}

De haber otro lento crecimiento de la economía mundial el 2012, se espera que China pueda otra vez usar su política fiscal y monetaria para dinamizar su demanda interna y así contrarrestar la caída en sus exportaciones. Si China sigue creciendo, podrá seguir comprando las materias primas (y otros bienes) de países como el Perú.

Gráfico 5. Evolución de la moneda china frente al dólar (enero 2007 a octubre 2011)

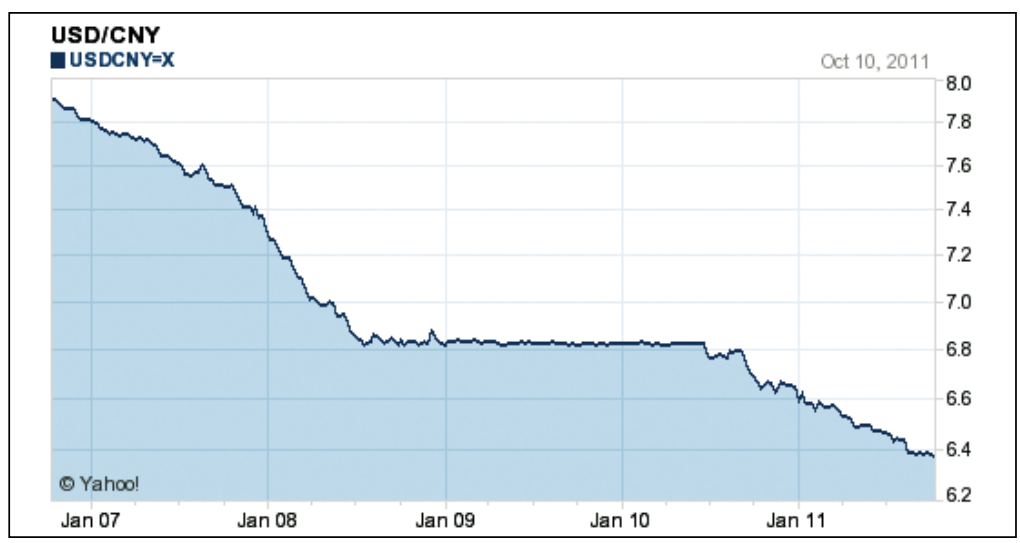

Fuente: http://finance. $y a h o o \cdot c o m / q / b c ? s=U S D C N Y=X \& t=5 y \& l=o n \& z=m \& q=1 \& c=$

De hecho, en el nuevo Plan Quinquenal que el gobierno chino ejecuta, el 12.', que va del 2011 al 2015, se planea que el consumo interno se convierta en el nuevo motor de la economía. La palabra que utilizan los chinos es rebalancear la economía, poniendo más énfasis en el consumo interno que en las exportaciones y la inversión. El mayor consumo podrá ser impulsado por la revaluación de su moneda, que hará más baratas las importaciones.

Por largos años, desde 1994, hasta julio del 2005, el yuan estaba en 8.2 yuanes por dólar y desde ese momento empezó a revaluarse hasta julio del 2008 en que quedó en 6.8 yuanes por dólar. No se movió casi desde este valor hasta julio del 2010 en que empezó a revaluarse otra vez y actualmente hasta octubre del 2011 estuvo en 6.38 yuanes por dólar. La tendencia es que el yuan se revalué, aunque muy lentamente y bajo la atenta mirada y el control de las autoridades chinas (Ver Gráfico 5). 


\section{Carlos Aquino Rodríguez}

Hay algunas dudas sobre la economía china, por ejemplo sobre las deudas malas que algunos de sus bancos han acumulado, algo que agencias como Fitch dice podría causar una rebaja en la calificación de su deuda 7 .

China de todas maneras se verá afectada por la desaceleración de la economía mundial originada en los países desarrollados, pues aun un $40 \%$ de sus exportaciones van a esos países, casi un $20 \%$ a EE.UU. y otro $20 \%$ a la Unión Europea. Hacia los países asiáticos va otro $40 \%$ aproximadamente ${ }^{8}$.

\section{Conclusiones}

China actualmente es el segundo mercado más grande del mundo, medido por el tamaño de sus importaciones totales, solo después de EE.UU. De haber otro lento crecimiento de la economía mundial el 2012, se espera que China pueda otra vez usar su política fiscal y monetaria para contrarrestar la caída en sus exportaciones. Si China sigue creciendo, podrá seguir comprando las materias primas (y otros bienes) de países como el Perú.

Hay que tomar en cuenta que China tiene 3.2 billones de dólares ${ }^{9}$ en reservas internacionales, que los puede usar en caso sea necesario y que su moneda, el yuan, se está revaluando, como referimos anteriormente. Esto aumenta el poder adquisitivo del país que puede comprar, así, más baratos productos del extranjero.

Se espera también que China aumente la importancia del consumo interno, y que este se convierta en el nuevo motor de su crecimiento económico. El consumo está en un nivel de 35\% de su PBI que es casi la mitad de muchos países. En el 2012, de haber una importante desaceleración de la economía mundial que haga que las exportaciones chinas caigan, el gobierno incentivará otra vez el consumo interno, como lo hizo en el 2009. De hecho ya se afirma que la mayor parte del crecimiento en China está viniendo de la inversión y consumo interno ${ }^{10}$.

7 http://www.bbc.co.uk/news/business-14836386

8 Ver Cuadro A11 Apéndice Estadístico: Asian Development Outlook 2011: South-South Economic Links, http://beta.adb.org/publications/asian-development-outlook-2011-south-south-economic-links

9 "Trade balance possible next year"

http://usa.chinadaily.com.cn/business/2011-08/23/content_13168604.htm

10 "China's economic growth slows to $9.1 \%$ in third quarter", http://www.bbc.co.uk/news/ business-15331523 


\section{Pensamiento Crítico N. ${ }^{\circ}$}

En todo caso, la influencia de China en el mundo seguirá creciendo. De hecho según informaciones China durante el 2009 y el 2010 prestó más dinero que el Banco Mundial a los países en desarrollo ${ }^{11}$. El Banco de Desarrollo de China y el EXIMBANK de China ofrecieron préstamos de por lo menos 110 mil millones de dólares, un $10 \%$ más de lo que el Banco Mundial prestó entre mediados del 2008 y mediados del 2010.

Para el Perú, como se ve en el Cuadro 6, China ya es el mayor mercado para nuestras exportaciones, pues en los primeros siete meses del año 2011 se vendió a China $25 \%$ más que a EE. UU., nuestro primer mercado por muchos años. En ese periodo el $15.5 \%$ de las exportaciones fueron a China y el $12.3 \%$ a EE. UU.

Cuadro 6. Exportaciones peruanas, enero-julio 2011

(valor fob, millones de dólares)

\begin{tabular}{|l|c|}
\hline \multicolumn{1}{|c|}{ Total } & $\mathbf{2 5 , 4 9 3 . 9 9 9}$ \\
\hline 1. China & $3,949.925$ \\
\hline 2. EE. UU. & $3,146.523$ \\
\hline 3. Suiza & $3,014.706$ \\
\hline 4. Canadá & $2,411.48$ \\
\hline 5. Japón & $1,338.9885$ \\
\hline 6. Chile & $1,172.890$ \\
\hline 7. Corea del Sur & $1,169.368$ \\
\hline
\end{tabular}

Fuente:http://www.aduanet.gob.pe/aduanas/informae/XRankPaisMensual_01072011.htm

Por lo general, China nos compra materias primas. Es nuestro principal comprador de hierro, harina de pescado, cobre, zinc y plomo. Las economías emergentes miran sobre todo a China, pues es el principal comprador de materias primas. El gigante oriental es una aspiradora de materias primas para alimentar su maquinaria industrial y para satisfacer las necesidades de sus consumidores, cada vez con más poder de compra. Como se ve en los Gráficos 6 y 7, entre 1999 y 2007, las importaciones chinas de soya y petróleo aumentaron en valor 35 veces, la de cobre en 25 veces, y la de hierro más de 7 veces (en volumen).

En todo caso, con las antiguas locomotoras del crecimiento mundial en problemas, el mundo mira a China ahora con gran expectativa.

11 http://www.bbc.co.uk/news/world-asia-pacific-12212936 


\section{Carlos Aquino Rodríguez}

Gráfico 6.

Demanda por

soya, petróleo y cobre en China

\section{Feeding frenzy}

China's imports of:

By value, January 1st 1999-100

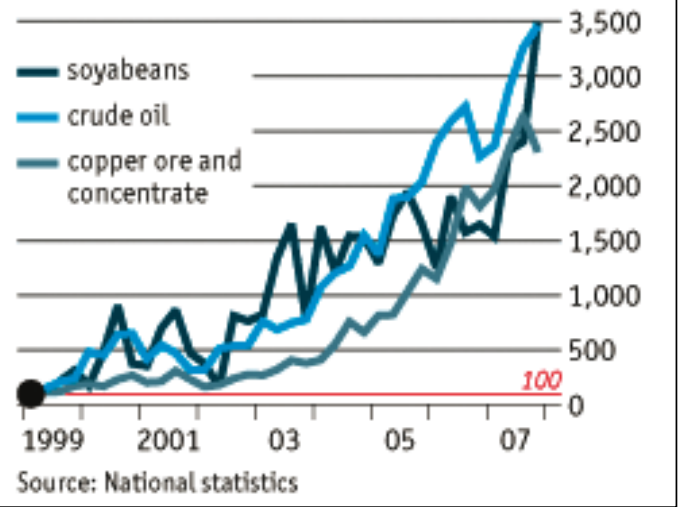

Fuente: "The Economist" Marzo 15-21, 2008

\section{Gráfico 7.}

Demanda por soya, petróleo y cobre en China

\section{The home front}

China's:

Tonnes, $\mathrm{m}$

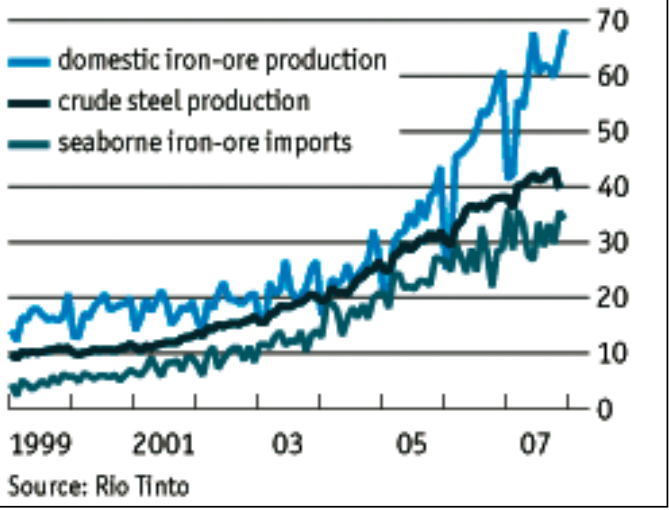

Fuente: "The Economist" Marzo 15-21, 2008 


\section{Bibliografía}

Asia development Bank: "Asian Development Outlook 2011: South-South Economic Links", abril 2011, http://beta.adb.org/publications/asian-development-outlook2011-south-south-economic-links

BBC News Asia, noticiero online: http:/www.bbc.co.uk/news/world/asia/

Diario online:"China Daily" varios numeros, http://usa.chinadaily.com.cn/index.html

Revista "The Economist" varios numeros 